\title{
Chronic obstructive pulmonary disease, bronchial asthma and allergic rhinitis in the adult population within the Commonwealth of Independent States: rationale and design of the CORE study
}

Yuriy Feshchenko', Liudmyla lashyna', Damilya Nugmanova², Olga Gyrina³, Maryna Polianska', Alexander Markov, Maryna Moibenko ${ }^{4}$, Janina Makarova ${ }^{5^{*}}$, Luqman Tariq ${ }^{6}$, Marcelo Horacio S. Pereira ${ }^{7}$, Eljan Mammadbayov ${ }^{8}$, Irada Akhundova ${ }^{8}$ and Averyan Vasylyev ${ }^{4}$

\begin{abstract}
Background: Main treatable Chronic Respiratory Diseases (CRDs) like Chronic Obstructive Pulmonary Disease (COPD), Bronchial Asthma (BA) and Allergic Rhinitis (AR) are underdiagnosed and undertreated worldwide. CORE study was aimed to assess the point prevalence of COPD, BA and AR in the adult population of major cities of Commonwealth of Independent States (CIS) countries - Azerbaijan, Kazakhstan, and Ukraine based on study questionnaires and/or spirometry, and to document risk factors, characterize the COPD, BA and AR population to provide a clearer "epidemiological data".

Methods: A descriptive, cross-sectional, population-based epidemiological study conducted from 2013 to 2015 with two-stage cluster geographical randomization. Interviewers conducted face-to-face visits at respondent's household after informed consent and eligibility assessment including interviews, anthropometry, spirometry (with bronchodilator test) and completion of disease-specific questionnaires.

Results: Two thousand eight hundred forty-two respondents (Ukraine: 964 from Ukraine; 945 from Kazakhstan; 933 Azerbaijan) were enrolled. Mean age was 40-42 years and males were 37\%-42\% across three countries. In Kazakhstan 62.8\% were Asians, but in Ukraine and in Azerbaijan 99.7\% and 100.0\%, respectively, were Caucasians. Manual labourers constituted 40.5\% in Ukraine, 22.8\% in Kazakhstan and 22.0\% in Azerbaijan, while office workers were 16.1\%, 31.6\% and 36.8\% respectively. 51.3\% respondents in Ukraine, 64.9\% in Kazakhstan and 69.7\% in Azerbaijan were married.
\end{abstract}

Conclusion: CORE study collected information that can be supportive for health policy decision makers in allocating healthcare resources in order to improve diagnosis and management of CRDs. The detailed findings will be described in future publications.

Trial registration: Study Protocol Summary is disclosed at GlaxoSmithKline Clinical Study Register on Jun 06, 2013, study ID 116757.

Keywords: Chronic respiratory diseases, COPD, Bronchial asthma, Allergic rhinitis, Prevalence, Study design, Risk factors, Ukraine, Kazakhstan, Azerbaijan

\footnotetext{
* Correspondence: yana.j.makarova@gsk.com

${ }^{5}$ GlaxoSmithKline, GSK Russia, Business Park "Krylatsky Hills", 17, Krylatskaya

Street, Building 3 ("Air"), 121614 Moscow, Russia

Full list of author information is available at the end of the article
} 


\section{Background}

The burden of chronic respiratory diseases (CRDs) is increasing over decades. Chronic Obstructive Pulmonary Disease (COPD), Bronchial Asthma (BA) and Allergic Rhinitis (AR) are the main treatable CRDs and the increase of mortality associated with these diseases is a clear indicator of late diagnosis and inadequate treatment $[1,2]$.

It became evident that the burden of CRDs is much higher than officially reported [3]. The prevalence of CRDs will be increasing, if CRD identification and management strategies are not changed, although studies show that major preventable CRDs can be adequately managed in developed [4] and developing countries [5], and even in deprived populations [6].

There is still a lack of knowledge related to CRDs prevalence in certain regions. Namely, little is known about the actual burden of CRDs in the Commonwealth of Independent States (CIS) area. The official governmental reports represent the diagnosed and treated cases but there is lack of data on the undiagnosed cases.

The first epidemiological study conducted in the Russian Federation as a part of Global Alliance Against Chronic Respiratory Disease (GARD) initiative [7] confirmed that CRDs are substantially underdiagnosed. This finding, as well as identified risk factors (high frequency of smoking [8], environmental pollution, occupational exposures [9], climatic peculiarities [10]) led to a conclusion that CRDs may represent a major healthcare problem for the Russian Federation and other CIS countries.

"Chronic Obstructive REspiratory diseases in CIS countries" (CORE) study was carried out to address the gap in the epidemiological estimate of the burden of main CRDs and their risk factors in the selected cities of the CIS region (in Ukraine, Kazakhstan and Azerbaijan).

Clinical protocol was worked out by GlaxoSmithKline (GSK) with participation and under advisory support of National Institute of Phthisiology and Pulmonology named after F.G. Yanovsky, National Academy of Medical Sciences of Ukraine (NIPP) and Semey State Medical University (Almaty, Kazakhstan). Spirometry trainings and assessment of quality and accuracy of lung function tests were carried out by NIPP.

This manuscript is the first in the series of publications devoted to the CORE study and is focused on describing the study design and baseline population characteristics.

\section{Methods}

\section{Study objectives}

The primary objective was to establish point prevalence of COPD, BA and AR in adult population in major cities of three CIS countries (Ukraine, Kazakhstan and Azerbaijan) based on American Thoracic Society (ATS) respiratory
Symptoms Questionnaire and spirometry. Study objectives are presented in Table 1.

\section{Study design}

CORE study is a descriptive, cross-sectional, multinational, population-based epidemiological study. A contract research organization (CRO) conducted the study in all countries. Independent Ethic Committee in Kazakhstan and Local Ethic Committees in all countries reviewed and approved the study. The study followed local data protection laws with respect of respondents and data confidentiality. The number of subjects recruited by country is shown in Fig. 1.

Before initiating the study, the CRO conducted feasibility study and selected the sites based on their ability to fulfil the study objectives (Table 2). General practitioners and/or pulmonologists, which manage CRDs, were selected as investigators.

The study was conducted from 2013 to 2015 (see Table 2). The majority of participants was enrolled during the period from July 2014 to October 2014 in Ukraine (599 participants), from June 2014 to September 2014 in Kazakhstan (676 participants), and from July 2015 to September 2015 in Azerbaijan (575 participants). Inclusion and exclusion criteria are summarised in Table 3.

Two-step cluster randomization (first step, administrative district; second step, street) was used for sampling strategy (Fig. 2). Investigators (interviewers) performed household visits to collect the data. Study Executive Committee (SEC) randomly selected streets for household visits in each city (country) applying stratified random cluster sampling procedure. The interviewers visited households sequentially, starting with the 1st apartment of the 1st number of the house in the selected street, and continuing in ascending order. Interviewers assessed eligibility of all inhabitants of every household.

In order to maintain adherence to the sampling strategy, in all cases when the potential participant was not available during the visit of interviewer, the interviewer performed up to three additional visits during different times of the day and during different weekdays (workdays and weekends) in order to try to identify the potential participant.

For any consented participant, all assessments (see Table 4) were expected to be completed in a single visit. In case of respondent's limited availability the interviewer scheduled another visit (up to three attempts) in order to complete interview and spirometry with that respondent.

The interviewer could perform additional visits to the participant in case of poor quality (in the opinion or Principal Investigator and/or SEC member) of spirometry results. 
Table 1 Study objectives

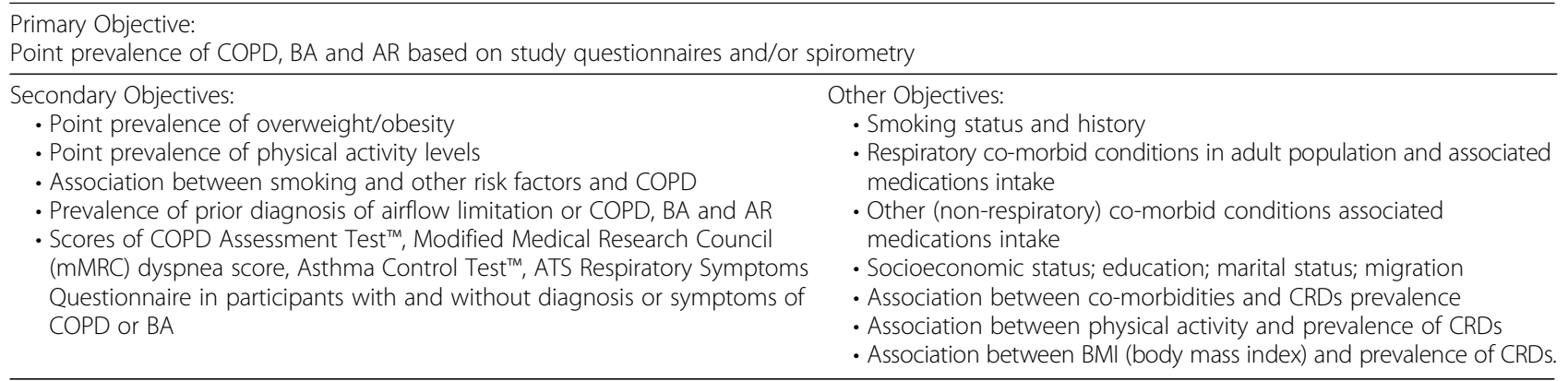

No treatment intervention, or follow up in these study participants, was conducted in this study. However, the investigator could contact the study participants with symptoms and/or spirometry abnormalities after the end of the study, in order to propose visit to a hospital to manage any potential airflow limitations or other symptoms identified during the study.

\section{Data collection}

Upon initial participants' agreement to proceed with the interview, the interviewer obtained their consent and assessed their eligibility to participate in the study using the questionnaires [11-15]. An additional file shows the questionnaires used in this study in more detail [see Additional file 1].

The interviewer measured the participant's height and body weight with a portable measuring device. All eligible participants provided general demographic information, medical history and recent use of medication and then underwent spirometry without bronchodilator (pre bronchodilator).

After this procedure, all participants were administered with the inhaled bronchodilator (salbutamol 200$400 \mathrm{mcg}$ ), and proceeded to the interview by answering the ATS Respiratory Symptoms Questionnaire (all respondents) [11], COPD Assessment Test (all respondents) [12] and Asthma Control Test ${ }^{\mathrm{Tm}}$ (respondents with previous diagnosis or asthma symptoms (Question 3B of ATS questionnaire)) [13]. By approximately 15$20 \mathrm{~min}$ after the first spirometry, all participants underwent the second spirometry testing (post bronchodilator).

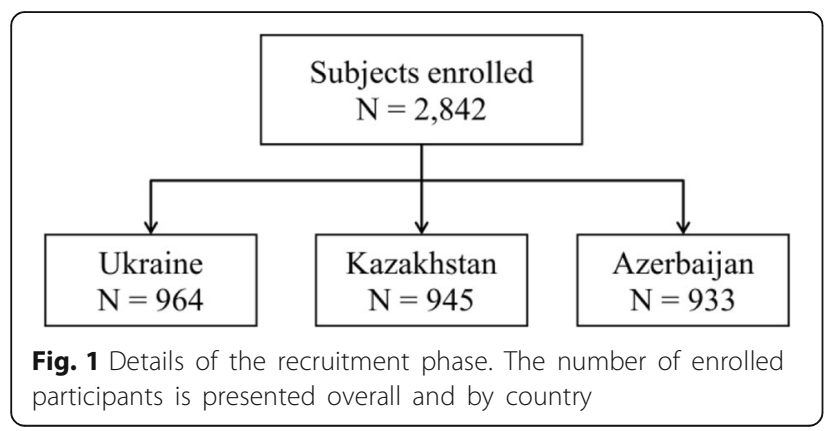

Participants were assessed by mMRC dyspnea score [14]. All participants answered the Alcohol Intake and Tobacco Smoking Questions as well as International Physical Activity Questionnaire [15]. See Additional file 1.

GlaxoSmithKline provided spirometry equipment (EasyOne $^{\mathrm{Tm}}$, ndd Medical Technologies, USA), height and body weight measuring devices, salbutamol MDIs (metered-dose inhalers), disposable spacers, gloves; a fully trained respiratory nurse or investigator operated all of them.

Diagnosis of COPD, BA and AR was established based on study questionnaires and/or spirometry (if applicable) using the following definitions:

COPD:

- Spirometry abnormalities alone based on criteria of GOLD (2011); [16]

- Previously diagnosed COPD self-reported by the respondent.

BA:

- Previously diagnosed BA self-reported by the participant;

- Presence of symptoms revealed by the ATS questionnaire (e.g. wheezing, cough, chest illness) with or without spirometry abnormalities. Wheezing (high-pitched whistling sounds) during the last 12 months is considered as BA-associated symptom for purposes of this study;

- Spirometry abnormalities alone (without symptoms revealed by the ATS questionnaire) should not be classified as BA, but classified as "BA-associated airflow limitation".

AR:

- Self-reported presence of watery runny nose symptom (during the last 12 months) alone or in combination with any of the following nasal or ocular symptoms: sneezing, nasal obstruction, nasal itching, or conjunctivitis. These symptoms will be revealed by Allergic Rhinitis Questionnaire [17, 18]. 
Table 2 Study sites

\begin{tabular}{|c|c|c|c|c|}
\hline Site \# & Country & City & Centre & Recruitment period \\
\hline 001 & Ukraine & Kiev & $\begin{array}{l}\text { National Medical University named after O.O. } \\
\text { Bogomolets, General Medicinal Department }\end{array}$ & 24 September 2013-26 October 2014 \\
\hline 002 & Kazakhstan & Almaty & Almaty Municipal Polyclinic \#2 & 09 December 2013-26 December 2014 \\
\hline 003 & Azerbaijan & Baku & Scientific Research Institute of Lung Diseases & 04 March 2015-01 November 2015 \\
\hline
\end{tabular}

- Presence of watery runny nose with one or more of the other nasal or ocular symptoms will suggest allergic rhinitis. Presence of watery runny nose alone suggests the respondent may have allergic rhinitis.

Investigators had to use Global Initiative for Asthma (GINA) [19], Global Initiative for Chronic Obstructive Lung Disease (GOLD) [16] and International Primary Care Respiratory Group (IPCRG) [17] guidelines to verify diagnosis of participants, based on available data.

\section{Statistical analysis}

Sample size was calculated using a design effect parameter to take account of possible heterogeneity between districts or squares in the stratified sampling strategy [20-22]. It was estimated that using 95\% confidence level, a design effect of 1.25 , a total sample of 465 individuals in each age group (930 in total) in each country would result in the following precision: prevalence of up to $10 \%$ with a margin of error no larger than $\pm 3.0 \%$; prevalence of up to $15-20 \%$ with a margin of error no larger than $\pm 4.0 \%$. Sample size calculation was made using the NCSS-PASS package (NCSS, LLC, USA) released on July 14, 2006.

Statistical analysis was performed using IBM SPSS Statistics software (IBM Corp., USA) version 21.0 and R software version 3.1.2 (R Core Team, Austria).

All analyses were performed using descriptive statistical methods. For the purpose of this manuscript, respondent characteristics will be provided as means (standard deviations) for continuous variables and as absolute frequencies (percentages) for categorical variables.

Essential data analysis will include:

1) Point prevalence of COPD (overall and separate stages)/BA/AR - as the number of COPD/BA/AR individuals divided by total number of subjects included in the study, and expressed as a number per 1000 for each country. Confidence intervals will be calculated for each frequency. The data obtained for prevalence of respiratory diseases will be extrapolated to other city population in the country.

2) Sex-adjusted, ethnicity-adjusted, age-adjusted point prevalence of COPD (overall and separate stages)/ BA/AR.

3) Age-adjusted (18-39, 40-64, above 65 years) prevalence of COPD (overall and separate stages)/ BA/AR.

4) Peak prevalence of COPD (overall and separate stages)/BA/AR for age-adjusted prevalence (every 5 years for the age range from 18 to 80 years).

The final analysis will be performed separately for each country.

\section{Results}

A total of 964 respondents in Ukraine, 945 respondents in Kazakhstan and 933 respondents in Azerbaijan were enrolled into the study meeting all the inclusion criteria (Fig. 1). 961 (Ukraine), 944 (Kazakhstan) and 933 (Azerbaijan) respondents completed the study according to the protocol (i.e. had all spirometry data available and questionnaires completed).

Baseline and demographic data were assessed for all respondents regardless of their completion of withdrawal status (Table 5).

Full-time employed respondents were predominant: Ukraine - 469 (48.7\%), Kazakhstan - 440 (46.6\%), Azerbaijan - 406 (43.5\%). In Ukraine 125 (13.0\%) were part-time employees, in Kazakhstan 178 (18.8\%) and in Azerbaijan - 174 (18.6\%) respondents were homemakers. In Ukraine the majority of respondents

Table 3 Inclusion and exclusion criteria

\begin{tabular}{|c|c|}
\hline Inclusion criteria & Exclusion criteria \\
\hline $\begin{array}{l}\text { - The informed consent for participation in the study has been } \\
\text { signed by the participant } \\
\text { - The participant's age from } 18 \text { years (inclusive) and above } \\
\text { - Willing to perform the spirometry and answer to the study } \\
\text { questionnaire } \\
\text { - } \geq 10 \text { year of residence in selected city according to confirmation } \\
\text { provided by participant }\end{array}$ & $\begin{array}{l}\text { - Any contraindication for lung function test, which may have potential harm } \\
\text { to participant based on a judgment of Investigator } \\
\text { - Age }<18 \text { years old } \\
\text { - Duration of permanent residence in selected study city <10 years } \\
\text { - Inability to perform the spirometry or respond to questionnaires } \\
\text { - Known hypersensitivity or contraindications to bronchodilator (salbutamol) } \\
\text { - Subjects with a pre-existing condition which, in the opinion of the investigator, } \\
\text { would compromise the safety of the subject in this study }\end{array}$ \\
\hline
\end{tabular}




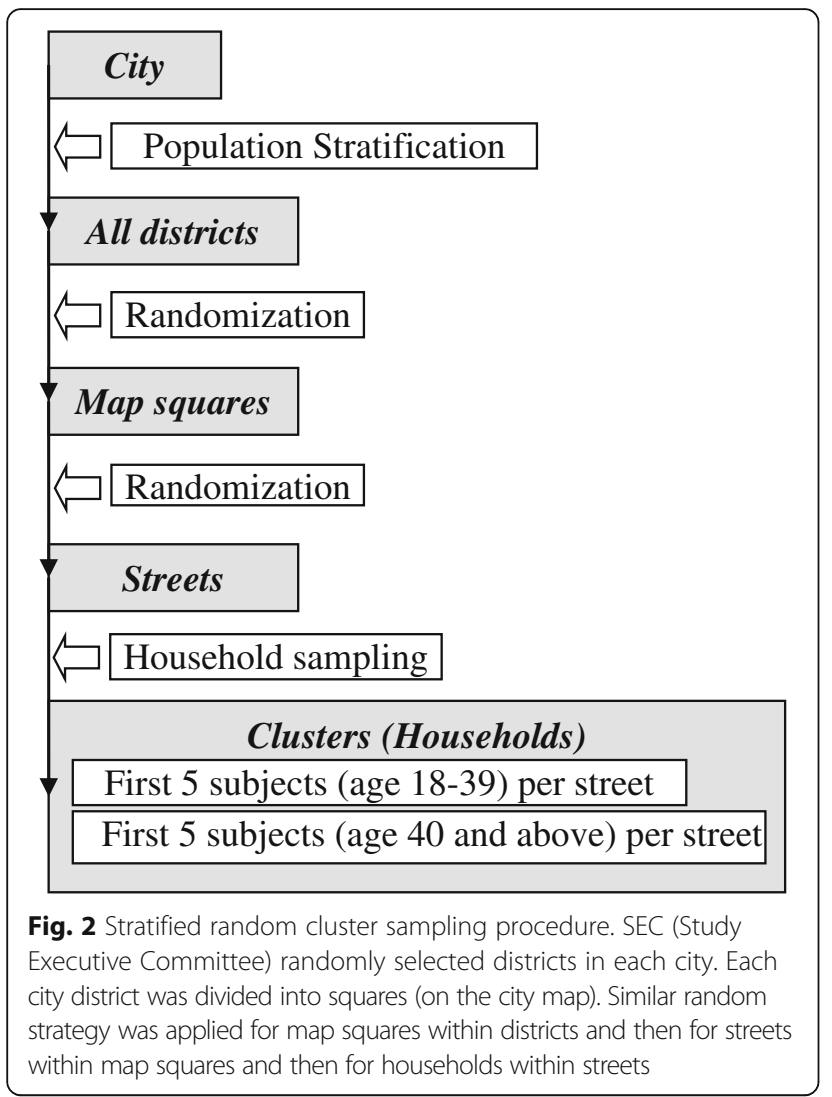

were manual labourers (390, 40.5\%), but not in Kazakhstan $(215,22.8 \%)$ or Azerbaijan (205, 22.0\%). Major part of the respondents $(308,32.6 \%)$ in Kazakhstan and $(347,37.2 \%)$ in Azerbaijan didn't report the type of activities. The second place in frequency was for "clerical / administrator" type of work (299 (31.6\%) in Kazakhstan and 343 (36.8\%) in Azerbaijan. An additional file shows distribution of the respondents by their employment status in more detail [see Additional file 2].

Respondents with bachelor's degree were predominant (276, 28.6\%) in Ukraine, while respondents with associate degree (occupational/technical/vocational program) were predominant $(257,27.2 \%)$ in Kazakhstan. The most frequent education status in Azerbaijan was professional school degree $(158,16.9 \%)$. An additional file shows distribution of the respondents by their education status in more detail [see Additional file 3].

Although more than one-half of sample was married in all countries, in Ukraine and Kazakhstan there was reasonably higher rate of singles in Ukraine (317, $32.9 \%$ ) compared to Kazakhstan $(195,20.6 \%)$ and to Azerbaijan (229, 24.5\%). An additional file shows distribution of the respondents by their marital status in more detail [see Additional file 4].
Table 4 Data collection flow chart

\begin{tabular}{|c|c|c|c|}
\hline Steps & Procedures & $\begin{array}{l}\text { Study visit } \\
\text { (Household visits) }\end{array}$ & $\begin{array}{l}\text { After study } \\
\text { visit }\end{array}$ \\
\hline 1. & Informed consent & $x$ & \\
\hline 2. & Assessment of eligibility criteria & $x$ & \\
\hline 3. & General demographic information & $x$ & \\
\hline 4. & $\begin{array}{l}\text { Body weight, height measurement } \\
\text { (using scales and rules) }\end{array}$ & $x$ & \\
\hline 5. & $\begin{array}{l}\text { Pre-dose spirometry (using portable } \\
\text { spirometer) }^{\mathrm{a}}\end{array}$ & $x$ & \\
\hline 6 & $\begin{array}{l}\text { Short-acting bronchodilator } \\
\text { inhalation via spacer }\end{array}$ & $x$ & \\
\hline 7. & $\begin{array}{l}\text { Medical history and concomitant } \\
\text { medications }\end{array}$ & $x$ & \\
\hline 8. & $\begin{array}{l}\text { ATS Respiratory Symptoms } \\
\text { Questionnaire }\end{array}$ & $x$ & \\
\hline 9. & questions & $x$ & \\
\hline 10. & $\begin{array}{l}\text { Post-dose spirometry (using } \\
\text { portable spirometer) }\end{array}$ & $x$ & \\
\hline 11. & Asthma Control Test ${ }^{\mathrm{TM}}$ & $x$ & \\
\hline 12. & COPD Assessment Test ${ }^{\mathrm{TM}}$ & $x$ & \\
\hline 13. & $\begin{array}{l}\text { Modified Medical Research } \\
\text { Council dyspnea score }\end{array}$ & $x$ & \\
\hline 14. & Alcohol intake questions & $x$ & \\
\hline 15. & Tobacco smoking & $x$ & \\
\hline 16. & $\begin{array}{l}\text { International Physical Activity } \\
\text { Questionnaire }\end{array}$ & $x$ & \\
\hline 17. & Adverse events assessment ${ }^{b}$ & $x$ & \\
\hline 18. & $\begin{array}{l}\text { Local spirometry quality review } \\
\text { (Site review) }\end{array}$ & & $x$ \\
\hline 19. & $\begin{array}{l}\text { Central spirometry quality review } \\
\text { (Central review) }\end{array}$ & & $x$ \\
\hline
\end{tabular}

aefore spirometry - body weight, height are to be measured, anamnesis and data of current medication (within previous 7 days) should be collected ${ }^{\mathrm{b}}$ Only Serious Adverse Events that the investigator considers related to study participation are to be recorded

\section{Discussion}

The CORE study aimed at assessing the point prevalence of COPD, BA and AR in the adult population of major cities of Azerbaijan (Baku), Kazakhstan (Almaty), and Ukraine (Kiev). Results provided in this publication demonstrated some differences between cities.

Although no formal statistical comparison was done between the three samples, it seems to be that Kazakhstan sample is relatively elder $(+2$ years $)$ and has more women $(+5 \%)$ compared to Ukraine and Azerbaijan. The most pronounced difference was in ethnicity: Asians are more than $60 \%$ in Kazakhstan, while in other countries almost $100 \%$ are Caucasians. Age, gender and ethnicity may constitute as confounding factors that should be taken into account during the analysis of CRDs prevalence and their risk factors.

GARD study [7] confirmed that occupational hazard is a major and independent risk factor for all CRDs. Thus, higher prevalence of manual labourers (40.5\%) in Ukraine 
Table 5 Demographic characteristics of sample respondents

\begin{tabular}{lllll}
\hline & & Ukraine & Kazakhstan & Azerbaijan \\
\hline Age (years) & Mean (SD) & $40.7(15.1)$ & $42.5(15.3)$ & $40.7(14.8)$ \\
Gender & Male & $403(41.8 \%)$ & $348(36.8 \%)$ & $389(41.7 \%)$ \\
& Female & $561(58.2 \%)$ & $597(63.2 \%)$ & $544(58.3 \%)$ \\
& Total & 964 & 945 & 933 \\
Ethnicity & Asian & $3(0.3 \%)$ & $593(62.8 \%)$ & 0 \\
& Black & 0 & $1(0.1 \%)$ & 0 \\
& Caucasian/White & $961(99.7 \%)$ & $349(36.9 \%)$ & $933(100.0 \%)$ \\
& Other & 0 & $2(0.2 \%)$ & 0 \\
& Total & 964 & 945 & 933 \\
BMl (kg/m²) & Mean (SD) & $25.0(5.1)$ & $25.7(5.1)$ & $26.4(5.3)$ \\
Weight category & BMl $<25 \mathrm{~kg} / \mathrm{m}^{2}$ & $526(54.6 \%)$ & $495(52.4 \%)$ & $419(45.1 \%)$ \\
& BMI $\geq 25 \mathrm{~kg} / \mathrm{m}^{2}$ & $437(45.4 \%)$ & $449(47.6 \%)$ & $511(54.9 \%)$ \\
& Total & 963 & 944 & 930 \\
Weight (kg) & Mean (SD) & $72.6(16.0)$ & $71.7(15.7)$ & $73.2(15.7)$ \\
Height (cm) & Mean (SD) & $170.1(9.2)$ & $167.0(8.6)$ & $166.4(8.6)$ \\
\hline
\end{tabular}

compared to Kazakh (22.8\%) and Azerbaijanian (22.0\%) samples with the opposite distribution of clerical/administration workers (16.1\% for Ukraine and 31.6\% and 36.8\% for Kazakhstan and Azerbaijan respectively) may affect the CRD-related estimates. Possible influence of educational background is not that evident, especially given that there is no direct correlation between education and employment area in the countries of former Soviet Union [23].

Finally, the proportion of married respondents in Kazakh and Azerbaijanian samples was about 13\% higher than in Ukraine; marital status may influence a CRD-related quality of life [24].

Study limitations should be taken into account during further analysis. Firstly, although two-level cluster randomization has been proven as an efficient approach when one-source population registry is not available [7], it may not ensure completely random sampling. Secondly, the study was conducted in urban population only (results may be not representative for rural area and the whole country).

Nevertheless, when compared to other epidemiology studies conducted in Ukraine [25, 26], Kazakhstan [23, 27] and Azerbaijan [26], CORE study shows a marked similarity in terms of sociodemographic parameters. In the absence of officially published demographic data in study countries, this fact probably reflects the correct sampling methodology.

It is important to note, the study involved independent quality review of lung function tests by the pulmonology specialists (Study Executive Committee) to ensure consistency of results in all study locations. This Committee performed all spirometry and questionnaire training and re-training, if required, for all Investigators, who performed spirometry measurements across all countries, ensuring consistency of investigational team knowledge and data, which we consider as an obvious methodological strength of this study.

There are also disease-related limitations: relatively low prevalence of COPD, BA and AR limits the subgroup analysis and assessment of association strength as well as treatment pattern analysis. Certain season fluctuations may influence the assessment of $\mathrm{BA}$ and $\mathrm{AR}$ symptoms due to hay fever. Although the recruitment was ongoing for a year, a portion of the participants were enrolled in a shorter (mainly summer time) period that might impact the assessment of CRDs symptoms.

There are also design-specific limitations intrinsic for any cross-sectional study [28], namely, with the lack of longitudinal follow up, this study will only be able to investigate associations, and will not be able to confirm causality in any significant associations detected.

Finally, it is widely recognised [2, 4, 5, 7], that study population across the countries may vary and such differences between the countries exist and need to be considered in the interpretation of the primary and secondary study endpoints.

\section{Conclusions}

CORE study collected and analysed information related to CRDs from the adult population of major cities in the CIS region. This valuable information can be supportive for health policy decision makers in allocating healthcare resources in order to improve their diagnosis and manage these CRDs. The detailed findings from the CORE study will be described in future publications.

\section{Additional files}

Additional file 1: "Study questionnaires used in the CORE study" contains description of the patient-reported questionnaires used in the CORE study. (DOCX 16 kb)

Additional file 2: "Employment status of the respondents in the CORE study" contains tabular data on distribution of the respondents by their employment status. (ZIP 23 kb)

Additional file 3: "Education status of the respondents in the CORE study" contains tabular data on distribution of the respondents by their education status. (ZIP $25 \mathrm{~kb}$ )

Additional file 4: "Marital status of the respondents in the CORE study" contains tabular data on distribution of the respondents by their marital status. (ZIP $19 \mathrm{~kb}$ )

Abbreviations

AR: Allergic rhinitis; ATS: American Thoracic Society; BA: Bronchial asthma; BMI: Body mass index; CIS: Commonwealth of Independent States; COPD: Chronic obstructive pulmonary disease; CRD: Chronic respiratory disease; CRO: Contract research organization; GARD: Global alliance against chronic respiratory diseases; GOLD: Global initiative for chronic obstructive lung disease; GSK: GlaxoSmithKline; mMRC: modified Medical Research Council; SEC: Study executive committee 


\section{Acknowledgments}

We thank all investigators for their contribution to the study, allowing the first clinical epidemiology research to happen with a high level of integrity and the contract research organisation, Synergy Research Group, which was funded by GlaxoSmithKline, performing the study organisation and conduct.

\section{Funding}

Funding for this study was provided by GSK (GSK Study Number RES116757, GSK study acronym: CORE). GSK was involved in the study design, collection, analysis, and interpretation of the data, in the writing of the report, and in the decision to submit the article for publication.

\section{Availability of data and materials}

The datasets used and/or analysed during the current study are available from the corresponding author on reasonable request.

\section{Authors' contributions}

All listed authors meet the criteria for authorship set forth by the International Committee for Medical Journal Editors. YF, LI, DN, MP, AV provided support in the study concept and protocol development, MP provided support in the spirometry quality review and study staff trainings. National Institute of Phthisiology and Pulmonology F.G. Yanovsky National Academy of Medical Sciences of Ukraine (NIPP), Kiev, Ukraine and Semey State Medical University, Almaty, Kazakhstan provided advisory support to the study. Editorial support in the form of draft outline, editorial suggestions to draft versions of this paper, collating author comments, assembling tables and figures, referencing, and copyediting was provided by Julia She at Synergy Research Group and was funded by GSK. YF, LI, DN, MP, AV, MM, AM, MH, JM, LT, OG, EM, IA took active part in this study design, acquisition of data, analysis and interpretation of the study data. YF, LI, DN, MP, AV, MM, AM, MH, JM, LT, OG, EM, IA participated in critical revisions of the manuscript and have approved the article for publication. The authors contributed to manuscript review, applying their clinical, epidemiology, and study design expertise and take responsibility for the integrity of the data and the accuracy of the data analysis.

\section{Ethics approval and consent to participate}

The study was reviewed and approved by Independent Ethic Committee in Kazakhstan (Central Commission for Ethics at the Ministry of Health of the Republic of Kazakhstan) and by Local Ethic Committees in Kazakhstan, Azerbaijan and Ukraine (Ethic Committee at Semey State Medical University, Almaty, Kazakhstan; Ethic Committee at Scientific Research Institute of Lung Diseases in Baku, Azerbaijan; Commission for Ethics at National Institute of Phthisiology and Pulmonology F.G. Yanovsky of NAMS, Kiev, Ukraine; Commission for Ethics at Center for Primary Health Care \#2 of Shevchenko District, Kiev, Ukraine), according to the local legal requirements. Written informed consent was obtained from each participant before any procedures or data collection related to the study.

\section{Consent for publication}

Not applicable.

\section{Competing interests}

The study was sponsored by GlaxoSmithKline (GSK) marketing a number of treatments for COPD, Allergic Rhinitis, and Asthma. DN, YF, MP, LY, OG, EM IA report grants from GSK, during the conduct of the study and personal fees from GlaxoSmithKline outside the submitted work. MM, JM are employees of GSK. AV and LT are GSK employees and shareholder. AM and MHSP were employees of GSK at the time this research was conducted.

\section{Publisher's Note}

Springer Nature remains neutral with regard to jurisdictional claims in published maps and institutional affiliations.

\section{Author details}

'National Institute of Phthisiology and Pulmonology F.G. Yanovsky of NAMS, Kiev, Ukraine. ${ }^{2}$ Semey State Medical University, Almaty, Kazakhstan. ${ }^{3}$ National Medical University n.a. O.O. Bogomolets, Kiev, Ukraine. ${ }^{4}$ GlaxoSmithKline, Kiev, Ukraine. ${ }^{5}$ GlaxoSmithKline, GSK Russia, Business Park "Krylatsky Hills", 17, Krylatskaya Street, Building 3 ("Air"), 121614 Moscow, Russia.

${ }^{6}$ GlaxoSmithKline, Dubai, UAE. ${ }^{7}$ Department of Internal Medicine, State
University of Rio de Janeiro (UERJ), Rio de Janeiro, Brazil. ${ }^{8}$ Scientific Research Institute of Lung Diseases, Baku, Azerbaijan.

Received: 17 February 2017 Accepted: 29 June 2017

Published online: 10 October 2017

\section{References}

1. World Health Organization. Global Surveillance, Prevention and Control of Chronic Respiratory Diseases. A Comprehensive Approach. 2007. http://www.who.int/gard/publications/GARD\%20Book\%202007.pdf. Accessed August 25, 2015.

2. $\mathrm{Xu} \mathrm{W}$, Collet JP, Shapiro S, et al. Negative impacts of unreported COPD exacerbations on health-related quality of life at 1 year. Eur Respir J. 2010; 35:1022-30

3. Laurendi G, Mele S, Centanni S, et al. Global alliance against chronic respiratory diseases in Italy (GARD-Italy): strategy and activities. Respir Med. 2012;106:1-8.

4. Haahtela T, Tuomisto LE, Pietinalho A, et al. A 10 year asthma programme in Finland: major change for the better. Thorax. 2006;61:663-70.

5. Fischer GB, Camargos PA, Mocelin HT. The burden of asthma in children: a Latin American perspective. Paediatr Respir Rev. 2005;6:8-13.

6. Cloutier MM, Hall CB, Wakefield DB, Bailit H. Use of asthma guidelines by primary care providers to reduce hospitalizations and emergency department visits in poor, minority, urban children. J Pediatr. 2005;146:591-7.

7. Chuchalin AG, Khaltaev N, Antonov NS, et al. Chronic respiratory diseases and risk factors in 12 regions of the Russian Federation. Int J Chron Obstruct Pulmon Dis. 2014;12(9):963-74.

8. Kamardina TV. Current status of smoking and possible solutions. Public health and disease prevention. 2004;5:46-58. Article in Russian

9. Shiryaeva O, Aasmoe L, Straume B, Bang BE. An analysis of the respiratory health status among seafarers in the Russian trawler and merchant fleets. Am J Ind Med. 2011;54:971-9.

10. Grumm RH. The Central European and Russian Heat Event of July-August 2010. Bull Amer Meteor Soc. 2011;92:1285-96. doi:10.1175/2011BAMS3174.1

11. Bellia V, Pistelli F, Giannini D, et al. Questionnaires, spirometry and PEF monitoring in epidemiological studies on elderly respiratory patients. Eur Respir J Suppl. 2003:40:21s-7s.

12. Jones PW, Harding G, Berry P, Wiklund I, Chen WH, Kline LN. Development and first validation of the COPD Assessment Test. Eur Respir J. 2009;34:648-54.

13. Schatz M, Sorkness CA, Li JT, et al. Asthma Control Test: reliability, validity, and responsiveness in patients not previously followed by asthma specialists. J Allergy Clin Immunol. 2006;117:549-56.

14. Ekici A, Bulcun E, Karakoc T, Senturk E, Ekici M. Factors Associated With Quality of Life in Subjects With Stable COPD. Respir Care. 2015;60:1585-91.

15. Craig $C L$, Marshall $A L$, Sjöström $M$, et al. International physical activity questionnaire: 12-country reliability and validity. Med Sci Sports Exerc. 2003;35:1381-95.

16. Global Initiative for Chronic Obstructive Lung Disease. 2011. Available: http://www.goldcopd.org. Accessed August 25, 2015.

17. Levy ML, Fletcher M, Price DB, Hausen T, Halbert RJ, Yawn BP. International Primary Care Respiratory Group (IPCRG) Guidelines: diagnosis of respiratory diseases in primary care. Prim Care Respir J. 2006;15:20-34.

18. Allergic Rhinitis And Its Impact on Asthma. 2007. Available: http://allergo. lyon.inserm.fr/ORL/8.4.2.ARIA_Rhinite_allergique.pdf. Accessed 25 Aug 2015.

19. Global Initiative for Asthma. 2012. Available: http://www.ginasthma.org/. Accessed August 25, 2015.

20. Machin D, Campbell M, Fayers PM, Pinol A. Sample Size Tables for Clinical Studies. 2nd ed. Wiley; 1997.

21. Desu MM, Raghavarao D. Sample Size Methodology. New York: Academic Press; 1990.

22. Hahn GJ, Meeker WQ. Statistical Intervals. New York: John Wiley \& Sons; 1991.

23. Bobak M, Murphy M, Rose R, Marmot M. Societal characteristics and health in the former communist countries of Central and Eastern Europe and the former Soviet Union: a multilevel analysis. J Epidemiol Community Health. 2007;61:990-6.

24. Weldam SW, Lammers JW, Decates RL, Schuurmans MJ, et al. Health Qual Life Outcomes. 2013;5(11):190.

25. Webb CP, Bromet EJ, Gluzman S, et al. Epidemiology of heavy alcohol use in Ukraine: findings from the world mental health survey. Alcohol Alcohol. 2005:40:327-35 
26. Stickley A, Koyanagi A, Roberts B, Leinsalu M, Goryakin Y, McKee M. Smoking status, nicotine dependence and happiness in nine countries of the former Soviet Union. Tob Control. 2015;24:190-7.

27. Supiyev A, Nurgozhin T, Zhumadilov Z, Sharman A, Marmot M, Bobak M. Levels and distribution of self-rated health in the Kazakh population: results from the Kazakhstan household health survey 2012. BMC Public Health. 2014;30(14):768.

28. Sedgwick P. Cross sectional studies: advantages and disadvantages. BMJ. 2014;348:g2276.

Submit your next manuscript to BioMed Central and we will help you at every step:

- We accept pre-submission inquiries

- Our selector tool helps you to find the most relevant journal

- We provide round the clock customer support

- Convenient online submission

- Thorough peer review

- Inclusion in PubMed and all major indexing services

- Maximum visibility for your research

Submit your manuscript at www.biomedcentral.com/submit
Biomed Central 